\title{
Baseline Trajectories of Heavy Drinking and their Effects on Post-Randomization Drinking in the COMBINE Study: Empirically Derived Predictors of Drinking Outcomes During Treatment
}

\author{
Ralitza Gueorguieva, $\mathrm{PhD}^{1}$, Ran $\mathrm{Wu}, \mathrm{MS}^{2}$, Dennis Donovan, $\mathrm{PhD}^{3}$, Bruce R. Rounsaville, \\ $\mathrm{PhD}^{2}$, David Couper, $\mathbf{P h D}^{4}$, John H. Krystal, $\mathbf{M D}^{2,5}$, and Stephanie S. O'Malley, $\mathbf{P h D}^{2}$ \\ ${ }^{1}$ Yale University Schools of Public Health and Medicine, 60 College St, New Haven, CT, USA \\ ${ }^{2}$ Department of Psychiatry, Yale University School of Medicine, 34 Park St, New Haven, CT, USA \\ ${ }^{3}$ Department of Psychiatry and Behavioral Sciences, School of Medicine, University of \\ Washington, 1107 NE $45^{\text {th }}$ Street, Suite 120, Seattle, WA, USA \\ ${ }^{4}$ Department of Biostatistics, The University of North Carolina at Chapel Hill, 137 E Franklin St, \\ Suite 400, Chapel Hill, NC, USA \\ ${ }^{5}$ VA Connecticut Healthcare System, 950 Campbell Avenue, West Haven, CT, USA
}

\section{Abstract}

The COMBINE Study sought to answer questions about the benefits of combining behavioral and pharmacological interventions (naltrexone and acamprosate) in alcohol-dependent patients. Our goals were to identify trajectories of heavy drinking prior to randomization in COMBINE, to characterize subjects in these trajectories, and to assess whether pre-randomization trajectories predict drinking outcomes. We analyzed daily indicators of heavy drinking in 90 days prior to randomization using a trajectory-based approach. Each subject was assigned to the most-likely pre-randomization heavy drinking trajectory, and the baseline characteristics of participants in the baseline trajectories were compared. Main and interactive effects of these trajectories and treatment factors (acamprosate, naltrexone or $\mathrm{CBI}$ ) on summary drinking measures during active

(C) 2011 Elsevier Inc. All rights reserved.

Corresponding author: Ralitza Gueorguieva, 60 College St., Room 215B, New Haven, CT 06520-8034, Tel: 203 974 7529, Fax: 203 974 7662, ralitza.gueorguieva@yale.edu.

Financial interests

Dr. Krystal has served as a scientific consultant and/or on the Scientific Advisory Board to the following companies: Abbot Laboratories, Aisling Capital, LLC, AstraZeneca Pharmaceuticals, Brint and Nicolini, Inc, Bristol-Myers Squibb, Easton Associates, Eli Lilly and Co, Gilead Sciences, Inc, GlaxoSmithKline, Janssen Pharmaceuticals, LoHocla Research Corporation, Lundbeck Research USA, Merz Pharmaceuticals, MK Medical Communications, Pfizer Pharmaceuticals, F. Hoffmann-La Roche Ltd, SK Holdings Co., Ltd, Takeda Industries, Teva Pharmaceutical Industries, Ltd and Transcept Pharmaceuticals. He is a co-sponsor for two patents under review for glutamatergic agents targeting the treatment of depression. He has the following patent: (1) Seibyl JP, Krystal $\mathrm{JH}$, Charney DS. Dopamine and noradrenergic reuptake inhibitors in treatment of schizophrenia.

Dr. Stephanie O'Malley: member American College of Neuropsychopharmacogy workgroup, the Alcohol Clinical Trial Initiative, sponsored by Abott Laboratories, Alkermes, Eli Lilly \& Company, GlaxoSmithKline, Johnson \& Johnson Pharmaceuticals, Lundbeck and Schering Plough; partner, Applied Behavioral Research; contract, Nabi Biopharmaceuticals; Advisory Board, Gilead Pharmaceuticals; consultant, Alkermes, GlaxoSmithKline, Brown University, University of Chicago, Scientific Panel of Advisors, Hazelden Foundation.

Publisher's Disclaimer: This is a PDF file of an unedited manuscript that has been accepted for publication. As a service to our customers we are providing this early version of the manuscript. The manuscript will undergo copyediting, typesetting, and review of the resulting proof before it is published in its final citable form. Please note that during the production process errors may be discovered which could affect the content, and all legal disclaimers that apply to the journal pertain. 
treatment (16 weeks) were assessed. We identified five trajectories of heavy drinking prerandomization: "T1: frequent heavy drinkers", "T2: very frequent heavy drinkers", "T3: nearly daily heavy drinkers", "T4: daily heavy drinkers" and "T5: daily heavy drinkers stopping early" prior to randomization. Trajectory membership was significantly associated with all drinking outcomes. Subjects in "T5: daily heavy drinkers stopping early" had comparable drinking outcomes to the subjects in "T1: frequent heavy drinkers" while the remaining trajectories were associated with significantly worse outcomes. Baseline trajectory did not interact significantly with treatment condition. These exploratory analyses confirmed the hypothesis that baseline trajectories predict post-randomization drinking outcomes. Interestingly, "T5: daily heavy drinkers stopping early" had outcomes that were comparable to the least severe baseline trajectory "T1: frequent heavy drinkers" and baseline trajectories of heavy drinking did not moderate treatment effects.

\section{Keywords}

trajectory-based analysis; clinical trial; baseline predictors; naltrexone; acamprosate; combined behavioral intervention

\section{Introduction}

The COMBINE study was designed to assess the effects of naltrexone, acamprosate and Combined Behavioral Intervention (CBI) in alcohol-dependent patients. The original analyses of the two primary endpoints, time to the first day of heavy drinking and percent days abstinent, revealed that either naltrexone or CBI without naltrexone improved outcomes (Anton et al, 2006) but the combination of CBI and naltrexone did not yield better outcomes than either treatment alone. No significant effects of acamprosate either alone or in combination were found.

Secondary trajectory-based analyses of pre-randomization any drinking days were performed to characterize the heterogeneity of participants' drinking behavior prior to entry into treatment with the goal of understanding some of the negative findings regarding acamprosate and the combination of naltrexone and CBI. In these analyses, we (Gueorguieva et al., 2011) identified five trajectories of any drinking in the 90 days prior to randomization and assessed predictive and moderating effects of these trajectories on postrandomization drinking outcomes. We showed that acamprosate appeared beneficial in a subset of the subjects (very frequent drinkers) and counterproductive in another subset (consistent daily drinkers who had longer duration of pretreatment abstinence: e.g. $\geq 14$ days). Baseline trajectories of any drinking also moderated naltrexone effects such that naltrexone improved outcome the most for very frequent drinkers. Baseline trajectories of any drinking did not moderate CBI effects.

Since baseline trajectories of any drinking assess only frequency of drinking and length of pre-randomization abstinence but not intensity of drinking, the goal of the current study was to extend the use of the trajectory-based approach to modeling baseline trajectories of heavy drinking which take these features into account. In our analyses of post-randomization trajectories of any drinking and heavy drinking in COMBINE (Gueorguieva et al., 2010) we found different numbers of trajectories for any and heavy drinking ( 6 for any drinking and 4 for heavy drinking) and differential treatment effects. For any drinking, CBI had a unique effect on reducing the likelihood of increasing to nearly daily drinking, naltrexone had a unique effect on decreasing nearly daily drinking, and the combination of naltrexone and $\mathrm{CBI}$ increased the chance of decreasing any drinking. For heavy drinking, each treatment alone (naltrexone, $\mathrm{CBI}$ ) and the combination lowered the chance to be in the consistent 
heavy drinkers trajectory but the combination was not better than either treatment alone. In contrast, in an analysis of data from VA study CSP425 of naltrexone (Gueorguieva et al., 2007), trajectories of any drinking and heavy drinking and treatment effects on these trajectories were very similar. Hence it is important to assess both trajectories of any and heavy drinking and it is likely that baseline trajectories of any and heavy drinking have different effects on outcomes.

In addition, heavy drinking is an important clinical measure providing further rationale for examining heavy drinking trajectories. Consensus panels conclude that percent heavy drinking days should be the primary outcome in alcoholism clinical trials (Allen, 2003) and the percent with no heavy drinking days (PNHDD) has been recommended as a dichotomous index of good response (Falk et al., 2010) that is associated with low risk of alcohol related consequences. Percent heavy drinking days also appears to be sensitive to the beneficial effects of naltrexone (Pettinatti et al., 2006).

We focused on daily measures of heavy drinking because they allowed us to capture changes in drinking more sensitively than measures of drinking summarized over longer time-periods (e.g., a week). While it would be of interest to directly model the number of drinks per day, these values have extreme distributions that can result in spurious latent classes (Bauer and Curran, 2003, 2004).

The main goals of the current study were to identify and characterize baseline trajectories of heavy drinking and to assess predictive and moderating effects of baseline trajectories of heavy drinking on post-randomization drinking outcomes. We focused on trajectory-based methods since these methods have proven useful in identifying developmental patterns of alcohol use (Muthén and Muthén, 2000a, b; Hill et al., 2000; Chassin et al., 2000; Del Boca et al., 2003; Greenbaum et al. 2004), in increasing power for assessment of treatment effects in clinical trials (Gueorguieva et al., 2007) and in revealing additional information about the moderators and potential mechanisms of treatment effects (Gueorguieva et al., 2010, Witkiewitz et al., 2007). Unlike standard summary outcomes, trajectories of heavy drinking capture different aspects of drinking: the frequency and the trend of intensive drinking and thus they can be regarded as composite measures of drinking. They also provide easily interpretable graphical summaries of patterns over time. The trajectory modeling strategy allows the data to guide the choice of the number of trajectories that best fit the data and to determine the shape of each trajectory over time. This approach utilizes all available data on each subject and can be used to estimate the proportion of the population whose treatment response corresponds most closely to each trajectory group.

Our a priori hypotheses were that baseline trajectories of heavy drinking will be similar to trajectories of any drinking with slightly smaller probabilities of membership in the more severe heavy drinking trajectories than in the corresponding any drinking trajectories. We also hypothesized that trajectories of heavy drinking days will prove to be an important predictor of treatment outcome independent of treatment condition since frequency of heavy drinking that exceeds nonhazardous limits ( 5 or more for men and 4 or more for women) is associated with increased risk of alcohol-related consequences (Breslow and Graubard, 2008). Based on our analyses of baseline trajectories of any drinking trajectories we were interested in testing the hypothesis that a trajectory associated with longer duration of pretreatment abstinence from heavy drinking will be associated with better outcomes than a trajectory with similar level of heavy drinking but shorter abstinence.

We expected that both naltrexone and acamprosate would have the strongest impact on drinkers whose baseline trajectories suggested a pattern of more persistent heavy drinking. These predictions were based on naltrexone's most consistent effect which is to reduce the 
frequency of heavy drinking (Pettinati et al., 2006) and acamprosate's hypothesized effect on attenuating protracted abstinence effects (De Witte et al., 2003; Heilig and Egli, 2006; Mann et al., 2008), which might be expected to be greater among more frequent heavy drinkers. However, motivated by our analyses of trajectories of any drinking, we were also interested in whether a trajectory of intermediate heavy drinking would be associated with a beneficial effect of acamprosate and a trajectory of daily heavy drinking with early initiation of abstinence with poorer outcomes with acamprosate. Since any drinking and heavy drinking capture different aspects of drinking behavior, we hypothesized that trajectories of any drinking and heavy drinking may have different effects on outcome. Thus, the current focus on trajectories of heavy drinking complements the assessment of the effects of prerandomization drinking trajectories.

\section{Materials and Methods}

\section{Participants}

The COMBINE study enrolled 1,383 subjects with alcohol dependence (Anton et al, 2006). Subjects were recruited by advertisements and from clinical referrals at 11 US academic sites between January 2001 and January 2004. Approximately 5000 subjects were screened, $72.1 \%$ did not meet eligibility criteria and were excluded, $27.9 \%$ (1383, 428 women and 955 men) were randomized. Participants' median age was 44 years, $71 \%$ had at least 12 years of education, and $42 \%$ were married. Ethnic minorities comprised $23 \%$ of the sample. Specific inclusion and exclusion criteria can be found in the main report (Anton et al., 2006). All subjects were required to have between 4 to 21 days of abstinence; and (3) more than 14 drinks (women) or 21 drinks (men) per week, with at least 2 heavy drinking days (defined as 4 or more drinks per day for women and 5 or more drinks per day for men) during a consecutive 30-day period within the 90 days prior to baseline evaluation.

\section{Procedures}

Eight groups received medication management (MM) and either placebos, naltrexone, acamprosate, or naltrexone + acamprosate. Half of these groups also received the CBI. A ninth group received CBI alone with no pills in order to examine placebo effects in secondary analyses.

\section{Measures}

Trained research assistants obtained daily report of drinking at baseline retrospectively using the Form 90 AIR-ED (Miller, 1996; Miller and DelBoca, 1994) and with the Timeline Follow-back interview (Sobell and Sobell, 1992, 1995) for the 16-week treatment period. Both are comprehensive self-report measures and have good reliability and internal consistency on summary drinking measures (Sobell and Sobell, 1992, 1995; Tonnigan et al, 1997). The dependent variable in our analysis was daily binary indicator of heavy drinking $(=1$ if four or more drinks were consumed by a female, or five or more drinkers were consumed by a male on a particular day, 0 otherwise).

The baseline characteristics were selected based on a priori expectations of their relevance to baseline heavy drinking trajectories or as predictors of outcome (Anton and Randall, 2005; Cisler et al., 2005). They also had complete data and meaningful statistical distributions. Drinking behavior variables derived from the Form 90 at baseline included peak BAC (averaged over the two heaviest drinking episodes in the 90 days prior to intake), drinks per drinking day, percent days abstinent, percent heavy drinking days and days of abstinence prior to randomization. Information about prior inpatient treatment or alcohol detoxification medications, legal history, and mental health problems was obtained on the Form90; history of alcohol withdrawal symptoms was obtained from the SCID-IV Alcohol Module (First et 
al., 1997); and current symptoms were obtained using the Clinical Withdrawal Assessment Scale- AR (Sullivan et al., 1989) administered at intake. Other clinical assessments included the Alcohol Dependence Scale (ADS; Skinner and Allen, 1982), the total score and the Impulsive Drinking Subscale of the Drinker Inventory of Consequences (DrInC; Miller et al., 1995), the total score of Obsessive Compulsive Drinking Scale (Anton et al., 1995). Commitment to Abstinence was determined from the treatment goal question from the Thoughts about Abstinence Scale (Hall et al., 1990). A binary variable was computed based on the response "I want to quit using alcohol once and for all, to be totally abstinent, and never use alcohol ever again for the rest of my life" versus all others. Participants completed these assessments at one of the intake appointments as described in the COMBINE methods paper (Combine Research Group, 2003).

Post-randomization drinking outcomes included percent days abstinent, percent heavy drinking days, good clinical outcome, abstinence from heavy drinking in last two months of the trial and continuous abstinence. Percent abstinent days and percent heavy drinking days were based on the available drinking data from randomization through week 16 . Good Clinical Outcome was defined as abstinence or moderate drinking without problems based on responses the Form 90 and DrInC. Moderate drinking was defined as a maximum of 11 (women) or 14 (men) drinks per week, with no more than 2 days on which more than 3 drinks (women) or 4 drinks (men) were consumed. Problems were defined as endorsing 3 or more items on the DrInC assessing physical, social, and psychological consequences of drinking. Abstinence from heavy drinking following a grace period has been proposed as an outcome measure for future clinical trials because it is associated with reduced risk of alcohol related consequences while allowing for improvements in drinking short of abstinence (Falk et al., 2010). We included continuous abstinence as an exploratory outcome because this was the primary outcome used in the approval of acamprosate by the FDA based on a reanalysis of data from three European clinical trials (Kranzler and Gage, 2008).

\section{Planned Analyses}

Identification of baseline heavy drinking trajectories-We used the approach of Nagin (1999) and Nagin and Tremblay (2001) to identify distinct trajectories of heavy drinking patterns during the 90 days prior to randomization. The models assumed fixed polynomial trends (linear, quadratic, cubic) over time within each trajectory. The final models were obtained via model selection (number of trajectory classes and degree of the polynomial trends over time) based on the Schwartz Bayesian criterion (BIC) and on having at least $5 \%$ of subjects in each trajectory class. For the analysis we used a customized SAS procedure (PROC TRAJ) developed by Jones et al (2001).

Based on the final trajectory models we calculated the posterior probabilities of membership in each trajectory class for each subject. We used categorical variables of trajectory class as response variables in the comparisons of baseline characteristics of the identified baseline trajectories and as predictors in the analyses of drinking outcomes during treatment. Classification accuracy was assessed using the entropy measure (Muthén, 2004) with values between 0.9 and 1 indicating excellent classification of individuals in trajectory classes.

Assessing the association between baseline any drinking and heavy drinking trajectories-We used bar plots to illustrate subjects' cross-classification in trajectories of any drinking (Gueorguieva et al., 2011) and trajectories of heavy drinking. We also calculated weighted kappa measure of agreement between the two classifications.

\section{Comparison of baseline heavy drinking trajectories on baseline characteristics-We used ANOVA and chi-square tests to compare baseline}


characteristics of the subjects classified in different trajectories. We also performed multivariate logistic regression and used backward elimination at the 0.05 level to determine which baseline variables were most strongly associated with trajectory membership and explained the largest proportion of variance in trajectory membership.

Predictive and moderating effects of baseline trajectories of heavy drinking on drinking outcomes-We modeled the effect of baseline heavy drinking trajectories and treatment (Naltrexone, Acamprosate, CBI and their interactions) on post-randomization drinking outcomes using general linear models for continuous variables and logistic regression models for binary outcomes.

For each drinking outcome, we performed backward elimination starting with a model with four-way and all lower order interactions among baseline trajectories, naltrexone, acamprosate and CBI. We then dropped non-significant interactions under the restriction that the model was hierarchically well formulated at each step and that the sample size for each combination of the categorical variables in the model was at least ten. All pair-wise mean differences were tested for statistical significance and odds ratios were calculated for the significant effects in the final models containing only main effects of baseline trajectories.

\section{Results}

\section{Identification of baseline heavy drinking trajectories}

Similar to our results for any drinking (Gueorguieva et al., 2011), we identified five distinct trajectories of heavy drinking prior to randomization (Figure 1). These trajectories can be referred to as

- "T1: frequent heavy drinkers",

- "T2: very frequent heavy drinkers"

- "T3: nearly daily heavy drinkers"

- “T4: daily heavy drinkers" and

- "T5: daily heavy drinkers stopping early".

Entropy of the model was excellent (0.97), thus most subjects were clearly assigned to a particular trajectory.

In all trajectories heavy drinking declined prior to randomization as required by the criteria for study entry; however, there were differences in the chance of heavy drinking over the 90 day baseline period and the abruptness with which drinking was discontinued prior to study entry. "T1: frequent heavy drinkers" ( $15.3 \%$ of the sample) had about $30 \%$ chance of drinking heavily on a particular day. "T2: very frequent heavy drinkers" ( $24.7 \%$ of the sample) had about $60 \%$ chance of drinking heavily on a particular day. "T3: nearly daily heavy drinkers" (21.0\% of the sample) had about $80 \%$ chance of drinking heavily on a particular day. "T4: daily heavy drinkers" ( $25.4 \%$ of the sample) had virtually $100 \%$ chance of drinking on a particular day and were barely able to stop drinking prior to study entry. In contrast "T5: daily heavy drinkers stopping early" (13.5\% of the sample) reduced their heavy drinking substantially at least 15 days in advance of randomization. This might reflect repeated attempts to achieve the required four consecutive days of abstinence prior to study entry. 


\section{Assessing the association between baseline any drinking and heavy drinking trajectories}

Figure 2 illustrates subjects' cross-classification in trajectories of any drinking and trajectories of heavy drinking. In Gueorguieva et al. (2011) we identified five trajectories of any drinking: "TA1: frequent drinkers" ( $40 \%$ chance of any drinking on a particular day), "TA2: very frequent drinkers" (75\% chance of drinking on a particular day), "TA3: nearly daily drinkers" (between 80 and $90 \%$ chance of drinking on a particular day), "TA4: consistent daily drinkers" (virtually 100\% chance of drinking on a particular day and barely able to stop drinking prior to study entry) and "TA5: daily drinkers abstaining early" (virtually $100 \%$ chance of drinking on a particular day but stopping about 15 days prior to randomization). The shapes of the trajectories of any drinking and heavy drinking were similar. Most of the subjects classified in the three most intensive any drinking categories (TA3-TA5) were also classified in the three most intensive heavy drinking categories (T3T5). The less frequent drinkers (in TA1 and TA2) were sometimes classified in more intensive heavy drinking trajectories suggesting potential sample selection of individuals with relatively infrequent but heavier drinking into the trial. In particular, most of the subjects classified in "TA2: very frequent drinkers" were classified in "T2: very frequent heavy drinkers" (62.1\% of TA2) but a sizeable proportion were classified in "T3: nearly daily heavy drinkers" (20.9\% of TA2). All subjects classified in "TA1: frequent drinkers" were either classified in "T1: frequent heavy drinkers" (58.7\% of TA1) or in "T2: very frequent heavy drinkers" ( $41 \%$ of TA1). Nevertheless, the agreement between classifications in any drinking and heavy drinking trajectories was very good (weighted kappa $=0.70,95 \%$ CI: $(0.67,0.73))$.

\section{Comparison of baseline heavy drinking trajectories on baseline characteristics}

Table 1 compares the five heavy drinking trajectories on summary measures of alcohol consumption derived from the Form 90. After backward elimination only percent heavy drinking days and days of abstinence prior to randomization were significantly associated with trajectory membership (both p-values $<0.0001$ ). The other variables in Table 1 , while significantly associated with trajectory membership when considered one at a time, were no longer significant in a multivariate analysis. Table 2 compares the trajectories on other baseline characteristics found significant after backward elimination for all five trajectories or for the comparison between "T4: daily heavy drinkers" and "T5: daily heavy drinkers stopping early". Although patterns of heavy drinking prior to establishing pre-treatment abstinence were relatively similar for the two daily heavy drinking trajectories ("T4: daily heavy drinkers" and "T5: daily heavy drinkers stopping early", Figure 1), there were a number of univariate differences in baseline characteristics between these two trajectories (Tables 1 and 2). Participants in "T5: daily heavy drinkers stopping early" were more likely to have higher ADS scores (OR=1.08, 95\% CI: $(1.04,1.12)$ ) and lower OCDS scores $(\mathrm{OR}=0.93,95 \% \mathrm{CI}:(0.90,0.96))$. They also had more mental health problems $(\mathrm{OR}=1.81$, $95 \%$ CI: $(1.15,2.85))$, greater commitment to abstinence (OR=1.81, 95\% CI: $(1.15,2.84))$ and attendance of Alcoholics Anonymous ( $\mathrm{OR}=2.45,95 \%$ CI: $(1.39,4.30)$ ) compared to "T4: daily heavy drinkers".

\section{Predictive effects of baseline trajectories of heavy drinking on drinking outcomes}

There were significant naltrexone by CBI interactions on good clinical outcome, percent drinking days and percent heavy drinking days consistent with the original findings ( $\mathrm{p}=0.01$, 0.03 and 0.03 respectively). Main effects of baseline trajectory were also significant for all outcomes (Figure 3 and Figure 4, all p-values <.01). Baseline trajectory did not interact significantly with treatment condition.

Figure 3 shows that "T3: nearly daily heavy drinkers" (Least Squares Mean (LSM) $=27.40$, $\mathrm{SE}=1.72$ ) and "T4: consistent daily heavy drinkers" (LSM=31.85, $\mathrm{SE}=1.57)$ had 
significantly higher percentage of drinking days than the remaining trajectories (all $\mathrm{p}<0.005$ ) during the 16 week treatment period. "T4: daily heavy drinkers" also had significantly higher percent heavy drinking days ( $\mathrm{LSM}=20.34, \mathrm{SE}=1.25)$ than all other trajectories (all p $<0.005$ ) except "T3: nearly daily heavy drinkers". "T3: nearly daily heavy drinkers" had significantly higher percent heavy drinking days (LSM=17.90, $\mathrm{SE}=1.37$ ) than "T1: frequent heavy drinkers" ( $\mathrm{LSM}=7.56, \mathrm{SE}=1.61, \mathrm{p}<.0001)$ and "T5: daily heavy drinkers stopping early" (LSM=11.30, $\mathrm{SE}=1.72, \mathrm{p}=0.003$ ) but not compared to "T2: very frequent heavy drinkers" (LSM=12.98, $\mathrm{SD}=1.26, \mathrm{p}=0.009)$.

Figure 4 shows that compared to subjects in the three intermediate trajectories subjects in "T5: daily heavy drinkers stopping early" had significantly higher chance for continuous abstinence $\mathrm{OR}=1.89,95 \% \mathrm{CI}:(1.22,2.93)$; $\mathrm{OR}=2.49,95 \% \mathrm{CI}:(1.55,3.98)$ and $\mathrm{OR}=2.20$, 95\% CI: $(1.41,3.43$ respectively) and abstinence from heavy drinking in the last two months of the study (OR=1.82, 95\% CI: $(1.22,2.70)$; $\mathrm{OR}=2.11,95 \% \mathrm{CI}:(1.40,3.19)$ and $\mathrm{OR}=1.93$, $95 \%$ CI: $(1.30,2.86)$ respectively). Subjects in the three intermediate trajectories (T2, T3 and T4) had significantly lower chance for good clinical outcome compared to "T1: frequent heavy drinkers" (OR=0.40, 95\% CI: $(0.24,0.68)$; $\mathrm{OR}=0.38,95 \% \mathrm{CI}:(0.22,0.66)$ and $\mathrm{OR}=0.29,95 \% \mathrm{CI}:(0.18,0.49)$ respectively) but were not significantly different from subjects in "T5: daily heavy drinkers stopping early" (all p>0.05). Overall, trajectories "T1: frequent heavy drinkers" and "T5: daily drinkers stopping early" had the best outcomes and the remaining three trajectories were ordered in severity of outcome.

\section{Discussion}

The principal finding from the current study was that pre-treatment trajectories with greater frequency of heavy drinking were associated with poorer treatment outcome unless accompanied by a self-imposed period of longer pre-treatment alcohol abstinence. Thus we confirmed the hypothesis that differing patterns or trajectories of heavy drinking, during the 90 days prior to individuals' randomization into treatment are significant predictors of subsequent post-randomization outcomes, whether measured by parameters of drinking or more broadly based clinical outcome. Consistent with prior work looking at any drinking behavior during the pre-randomization period, the present analyses identified five specific patterns of heavy drinking. There was a high degree of overlap between the five heavy drinking trajectories with those previously found for any drinking, especially among the more frequent heavier drinking categories. That is, individuals who drank more frequently were also more likely to also be more frequent heavy drinkers as well.

Our results suggest that initiation of abstinence prior to treatment varied the most among daily heavy drinkers (subjects in $\mathrm{T} 4$ and $\mathrm{T} 5$ considered together) as compared to less frequent heavy drinkers (subjects in T1-T3). Stopping early among daily heavy drinkers may be partially due to motivation (commitment to abstinence in the "T5: daily heavy drinkers stopping early" was higher than in the other trajectories) but it may also be associated with interventions used to initiate abstinence (e.g. subjects who stop early are more likely to have received inpatient treatment).

While duration of abstinence from heavy drinking prior to treatment and frequency of heavy drinking are by themselves important predictors of outcome (Karim et al., 2010; McLellan et al., 1994; Moos and Moos, 2006), the value of trajectory-based analysis is that it allows simultaneous consideration of both aspects of heavy drinking in a data-driven fashion. Trajectory analyses capture simultaneously different aspects of drinking behavior and present an easily interpretable graphical summary of the patterns of drinking over time. Thus they provide advantages over summary drinking measures in identifying combinations of features of drinking that are associated with good outcome. 
The better outcomes seen for daily heavy drinkers who had established a period of abstinence are consistent with the results of Project Match, in which patients recruited into the aftercare arm of this study had higher percentage of days abstinent and lower drinks per drinking day than those recruited into the outpatient arm of the study (Project Match, 1998). Like Project Match, it is difficult to determine whether the better prognosis of those with extended pretreatment abstinence is due to baseline differences in the characteristics of participants, prior treatment experiences, or as a function of the abstinent period.

In examining baseline characteristics, the group with longer pre-treatment abstinence had the highest scores on a number of variables that would lead one to predict a poor prognosis relative to other trajectory groups. In particular, its members had the highest scores on measures related to chronicity and alcohol dependence (e.g. prior detoxification episodes, prior treatment, alcohol dependence symptoms, and negative consequences associated with drinking and alcohol dependence). However, in contrast to this clinical picture, or possibly because of it, the "T5: daily heavy drinkers stopping early" also had the greatest commitment to abstinence as a goal. This goal, as well as their greater involvement in prior treatment, may have in fact been used by group members to attain abstinence earlier in the period prior to randomization compared to the other group of heavy daily drinkers who did not stop early. Stopping early is likely to be the clearest behavioral marker of this commitment and the capacity to act on this. It is of note that the other daily heavy drinking trajectory group (T4), while having somewhat lower levels of predictors of poor outcome had the lowest proportion highly committed to abstinence.

The relevance of the commitment to abstinence between these two daily heavy drinking trajectories was manifested in the differences in post-randomization drinking outcomes. Interestingly, "daily heavy drinkers stopping early" (T5) had outcomes that were relatively comparable to and did not differ significantly from the outcomes of the least severe baseline trajectory "frequent heavy drinkers" (T1) while the other trajectories were associated with significantly worse outcomes. The outcomes of these latter groups were ordered in a manner consistent with the frequency of their pre-randomization heavy drinking, with the daily heavy drinking trajectory members (T4) having the highest percentages of drinking and heavy drinking and the lowest percentage of good clinical outcomes.

While there was a high correspondence of membership between individuals in the any drinking and heavy drinking trajectories, there were some substantial differences (e.g. less frequent drinkers were sometimes classified in more severe heavy drinking trajectories, and some daily heavy drinkers stopping early (T5) were classified into other any drinking trajectories which did not stop all drinking early). These two differences may explain why baseline trajectories of any drinking significantly moderated the effects of acamprosate but the same effect was not observed for baseline trajectories of heavy drinking. In our previous study of patterns of the frequency of any pre-randomization drinking (Gueorguieva, 2011) acamprosate was found to benefit those who were very frequent but not daily drinkers and appeared not to be indicated for patients who have already achieved an extended period of pre-treatment abstinence (e.g., >14 days). In the current analysis of heavy drinking, however, there were no differential benefits from acamprosate. This suggests that frequent drinking but not necessarily frequent heavy drinking predicts good outcome on acamprosate. In addition, early discontinuation of heavy drinking is not as informative as early cessation of all drinking for identifying individuals for whom acamprosate may not be indicated. What appear to be more predictive of acamprosate response are patterns of drinking days irrespective of the intensity of drinking. We were not able to test this potential explanation of the observed differences in acamprosate effects on any and heavy drinking as only a few combinations of trajectories of any and heavy drinking had sufficient sample sizes for meaningful inference. Alternative approaches integrating information on the occurrence and 
amount of drinking (e.g. directly characterizing the distribution of drinking data) may improve clinical prediction. However, this is still a very challenging issue as drinking data are both zero inflated and right-skewed and do not satisfy any standard statistical distribution. External validation of the results for any and heavy drinking in a different data set can also help better understand or resolve the differences in findings.

Similarly, naltrexone condition did not interact with baseline trajectories of heavy drinking although the interaction of naltrexone and CBI was significant on several outcomes, similar to the primary findings of COMBINE. The potential influence of pretreatment abstinence from heavy drinking in predicting response to naltrexone may have been attenuated because all patients in COMBINE, like most studies of oral naltrexone, were required to have at least 4 days of abstinence prior to randomization. In contrast, pretreatment abstinence was a predictor of stronger response to naltrexone in a trial of extended release naltrexone that did not require pretreatment abstinence (O'Malley et al., 2007; Garbutt et al., 2005).

The present findings have implications for both research and clinical practice. From a research perspective, data on baseline trajectory of heavy drinking could be used to inform expected response rates for planning clinical trials based on potential inclusion criteria related to heavy drinking. Clinically, the data indicate that individuals who are daily heavy drinkers but who are capable of achieving and maintaining a period of abstinence from heavy drinking have a good prognosis that exceeds what might be predicted from their clinical history. This is particularly likely in comparison to individuals who are daily heavy drinkers who only achieve a short duration of abstinence prior to treatment entry. The data suggest that patients who drink at similar levels may not be similar with respect to their capacity to respond to treatment or to modify their drinking. To better understand this readiness for treatment, we may need to build in self-enforced pre-treatment abstinence periods or other steps to reveal the latent capacity for change. Individuals in less favorable trajectories may benefit from early intervention with treatments such as contingency management (Petry et al., 2000; Carroll and Rounsaville, 2007) to promote abstinence and to transition into more favorable trajectories.

Our study has several limitations. First, since the COMBINE Study had strict inclusion/ exclusion criteria and study procedures, the results may not generalize to more complicated patients or to treatment provided in more general practice settings. Second, our analyses are exporatory and limited to the 16 weeks of treatment. External validation in a different sample is needed. Third, because of the large number of post-hoc tests, it is possible that type I error may be inflated. Fourth, the trajectory-based analyses are predicated on the assumption that different trajectory classes exist. If the population is homogeneous, then spurious findings may result.

Given the lack of interactions between the baseline heavy drinking trajectories and the pharmacological and behavioral therapies used in the COMBINE Study, the current results do not provide guidance about patient treatment matching. The discrepancy between our results for any drinking and heavy drinking may be due to the nature of the drinking measures: while any drinking captures frequency of drinking, heavy drinking is more focused on intensity of drinking. The analyses suggest that frequency of drinking is a significant moderator of acamprosate effects, but intensity of drinking is not a significant moderator of the effect of any treatment. Thus, for matching patients to treatments, trajectories of any drinking may be more informative.

\section{Acknowledgments}

This work was supported by the National Institute on Alcohol Abuse and Alcoholism [R01AA017173, P50 AA-012870, K05 AA014715, KO5 AA14906], the National Institute on Drug Abuse [P50 DA09241, K05 
DA00089], the National Center for Research Resources [NCRR UL1RR024139], the State of Connecticut, Department of Mental Health and Addiction Services, and the following U.S. Veterans Administration Centers: Mental Illness Research, Education and Clinical Center; Alcohol Research Center; Merit Review Program and National Center for PTSD.. The content is solely the responsibility of the authors and does not necessarily represent the official views of the funding agencies.

\section{References}

Allen JP. Measuring outcome in interventions for alcohol dependence and problem drinking: executive summary of a conference sponsored by the National Institute on Alcohol Abue and Alcoholism. Alcohol Clin Exp Res. 2003; 27:1657-1660. [PubMed: 14574237]

Anton RF, Moak DH, Latham P. The Obsessive Compulsive Drinking Scale: A self rated instrument for the quantification of thoughts about alcohol and drinking behavior. Alcohol Clin Exp Res. 1995; 19:92-99. [PubMed: 7771669]

Anton RF, O'Malley SS, Ciraulo D, et al. Combined pharmacotherapies and behavioral interventions for alcohol dependence. The COMBINE study: a randomized controlled trial. JAMA. 2006; 295:2003-2017. [PubMed: 16670409]

Anton RF, Randall CL. Measurement and choice of drinking outcome variables in the COMBINE Study. J Stud Alc Suppl. 2005; 15:104-109.

Bauer DJ, Curran PJ. Distributional assumptions of growth mixture models: Implications for overextraction of latent trajectory classes. Psychological Methods. 2003; 8:338-363. [PubMed: 14596495]

Bauer DJ, Curran PJ. The integration of continuous and discrete latent variable models: Potential problems and promising opportunities. Psychological Methods. 2004; 9:3-29. [PubMed: 15053717]

Breslow RA, Graubard BI. Prospective study of alcohol consumption in the United States: quantity, frequency, and cause -specific mortality. Alcohol Clin Exp Res. 2008; 32:513-521. [PubMed: 18215212]

Carroll KM, Rounsaville BJ. A perfect platform: combining contingency management with medications for drug abuse. Am J Drug Alcohol Abuse. 2007; 33:343-65. [PubMed: 17613963]

Chassin L, Pitts SC, Prost J. Binge drinking trajectories from adolescence to emerging adulthood in a high risk sample: predictors and substance abuse outcomes. J Consult Clin Psychol. 2002; 70(1):6778. [PubMed: 11860058]

Cisler RA, Kivlahan DR, Donovan D, Mattson ME. Assessing nondrinking outcomes in combined pharmacotherapy and psychotherapy clinical trials for the treatment of alcohol dependence. $\mathbf{J}$ Studies Alcohol Suppl. 2005; 15:110-118.

The COMBINE Study Research Group. Testing combined pharmacotherapies and behavioral interventions in alcohol dependence: Rationale and methods. Alcohol Clin Exp Res. 2003; 27:1107-1122. [PubMed: 12878917]

Del Boca FK, Darkes J, Greenbaum PE, Goldman MS. Up close and personal: temporal variability in the drinking of individual college students during their first year. J Consult Clin Psychol. 2003; 72(2):155-164. [PubMed: 15065951]

De Witte P, Pinto E, Ansseau M, Verbanck P. Alcohol and withdrawal: from animal research to clinical issues. Neurosci Biobehav Rev. 2003; 27:189-197. [PubMed: 12788332]

Falk D, Wang XQ, Liu L, Fertig J, Mattson M, Ryan M, Johnson B, Stout R, Litten RZ. Percentage of subjects with no heavy drinking days: evaluation as an efficacy endpoint for alcohol clinical trials. Alcohol Clin Exp Res. 2010; 34:2022-2034. [PubMed: 20659066]

First, MB.; Spitzer, RL.; Gibbon, M.; Williams, JBW. Structured Clinical Interview for DSM-IV Axis I Disorders-Clinician Version (SCID-CV). American Psychiatric Press; Washington, DC: 1997.

Garbutt JC, Kranzler HR, O’Malley SS, Gastfriend DR, Pettinati HM, Silverman BL, Loewy JW, Ehrich EW. Vivitrex Study Group. Efficacy and tolerability of long-acting injectable naltrexone for alcohol dependence: A randomized controlled trial. JAMA. 2005; 293 (13):1617-1625. [PubMed: 15811981]

Greenbaum PE, Del Boca FK, Darkes J, Wang CP, Goldman MS. Variation in the drinking trajectories of freshmen college students. J Consult Clin Psychol. 2004; 73(2):229-238. [PubMed: 15796630] 
Gueorguieva R, Wu R, Pittman B, Cramer J, Rosenheck R, O’Malley S, Krystal J. New Insights into the Efficacy of Naltrexone Based on Trajectory-Based Reanalyses of Two Negative Clinical Trials. Biol Psychiatry. 2007; 61:1290-1295. [PubMed: 17224132]

Gueorguieva R, Wu R, Donnovan D, Rounsaville B, Couper D, Krystal J, O’Malley S. Naltrexone and combined behavioral intervention effects on trajectories of drinking in the COMBINE study. Drug Alcohol Depend. 2010; 107:221-229. [PubMed: 19969427]

Gueorguieva R, Wu R, Donovan D, Rounsaville B, Couper D, Krystal J, O’Malley S. Baseline Trajectories of Drinking Moderate Acamprosate and Naltrexone Effects in the COMBINE study. Alcohol Clin Exp Res. 2011; 35(3):523-531. [PubMed: 21143249]

Hall SM, Havassy BE, Wasserman DA. Commitment to abstinence and acute stress in relapse to alcohol, opiates, and nicotine. J Consult Clin Psychol. 1990; 58:175-181. [PubMed: 2335634]

Heilig M, Egli M. Pharmacological treatment of alcohol dependence. Target symptoms and target mechanisms. Pharmacol Therapeut. 2006; 111:855-876.

Hill KG, White HR, Chung IJ, Hawkins JD, Catalano RF. Early adult outcomes of adolescent binge drinking: person and variable-centered analyses of binge drinking trajectories. Alcohol Clin Exp Res. 2000; 24(6):892-901. [PubMed: 10888080]

Jones B, Nagin DS, Roeder K. A SAS procedure based on mixture models for estimating developmental trajectories. Sociol Methods Res. 2001; 29:374-393.

Karim Z, Intaraprasong P, Scudamore CH, Erb SR, Soos JG, Cheung E, Cooper P, Buzckowski AK, Chung SW, Steinbrecher UP, Yoshida EM. Predictors of relapse to significant alcohol drinking after liver transplantation. Can J Gastroenterol. 2010; 24:245-250. [PubMed: 20431813]

Kranzler H, Gage G. Acamprosate efficacy in alcohol-dependent patients: Summary of results from three pivotal trials. American Journal of Addictions. 2008; 717:70-76.

Mann K, Kiefer F, Spanagel R, Littleton J. Acamprosate: Recent findings and future research directions. Alcohol Clin Exp Res. 2008; 32:1105-1110. [PubMed: 18540918]

Mason BJ, Crean R. Acamprosate in the treatment of alcohol dependence: clinical and economic considerations. Expert Review Neurotherapeutics. 2007; 7(11):1465-1477.

McLellan AT, Alterman AI, Metzger DS, Grissom GR, Woody GE, Luborsky L, O’Brien CP. Similarity of outcome predictors across opiate, cocaine, and alcohol treatments: Role of treatment services. J Consult Clin Psychol. 1994; 62:1141-1158. [PubMed: 7860812]

Miller WR, Del Boca FK. Measurement of drinking behavior using the Form 90 family of instruments. J Stud Alcohol Suppl. 1994; 12:112-118. [PubMed: 7722987]

Miller, WR.; Tonigan, JS.; Longabaugh, R. Test manual. National Institute on Alcohol Abuse and Alcoholism; Rockville, MD: 1995. Drinker Inventory of Consequences (DrInC): An instrument for assessing adverse consequences of alcohol abuse.

Miller, WR. NIAAA Project MATCH Monograph Series. Washington: Government Printing Office; 1996. Form 90: A structured assessment interview for drinking and related behaviors.

Moos RH, Moos BS. Rates and predictors of relapse after natural and untreated remission from alcohol use disorders. Addiction. 2006; 101:212-222. [PubMed: 16445550]

Muthén, BO. Mplus Technical Appendices. Los Angeles, CA: Muthén and Muthén; 2004. Appendix 8

Muthén BO, Muthén LK. Integrating person-centered and variable-centered analyses: Growth mixture modeling with latent trajectory classes. Alcohol Clin Exp Res. 2000a; 24(6):882-891.

Muthén BO, Muthén LK. The development of heavy drinking and alcohol-related problems from ages 18 to 37 in a U.S. national sample. J Stud Alcohol. 2000b:290-300.

Nagin DS. Analyzing developmental trajectories: a semiparametric, group-based approach. Psychol Methods. 1999; 4:139-157.

Nagin DS, Tremblay RE. Analyzing developmental trajectories of distinct but related behaviors: a group-based method. Psychol Methods. 2001; 6:18-34. [PubMed: 11285809]

O’Malley SS, Garbutt JC, Gastfriend DR, Dong Q, Kranzler HR. Efficacy of Extended-Release Naltrexone in alcohol-dependent patients who are abstinent prior to treatment. J Clin Psychopharm. 2007; 27(5):507-512. 
Petry NM, Martin B, Cooney JL, Kranzler HR. Give them prizes, and the will come: contingency management for treatment of alcohol dependence. J Consult Clin Psychol. 2000; 68:250-257. [PubMed: 10780125]

Pettinati HM, O’Brien CP, Rabinowitz AR, Wortman SP, Oslin DW, Kampman KM, Dackis CA. The status of naltrexone in the treatment of alcohol dependence: Specific effects on heavy drinking. $J$ Clin Psychopharm. 2006; 26(6):610-625.

Project Match Group. Matching alcoholism treatments to client heterogeneity: treatment main effects and matching effects on drinking during treatment. Project MATCH Research Group. J Stud Alcohol. 1998; 59(6):631-639. [PubMed: 9811084]

Skinner HA, Allen BA. Alcohol dependence syndrome: Measurement and validation. J Abnorm Psychol. 1982; 91:199 -209. [PubMed: 7096790]

Sobell, LC.; Sobell, MB. Timeline Follow-back: A technique for assessing self-reported ethanol consumption. In: Allen, J.; Litten, RZ., editors. Measuring alcohol consumption: Psychosocial and biological methods. New Jersey: Human Press; 1992.

Sobell, LC.; Sobell, MB. Alcohol consumption measures. In: Allen, JP.; Columbus, M., editors. Assessing alcohol problems: A guide for clinician and researchers. Rockville, MD: National Institute on Alcohol Abuse and Alcoholism; 1995. p. 55-73.

Sullivan JT, Sykora K, Schneiderman J, Naranjo CA, Sellers EM. Assessment of alcohol withdrawal: The revised Clinical Institute Withdrawal Assessment for Alcohol scale (CIWA-AR). Br J Addict. 1989; 84:1353-1357. [PubMed: 2597811]

Tonigan JS, Miller WR, Brown JM. The reliability of Form 90: An instrument for assessing alcohol treatment outcome. J Stud Alcohol. 1997; 58:358-364. [PubMed: 9203116]

Witkiewitz K, van der Maas HJ, Hufford MR, Marlatt GA. Non-normality and Divergence in Post Treatment Alcohol Use: Re-examining the Project MATCH Data “Another Way”. J Ab Psychol. 2007; 116(2):378-394. 


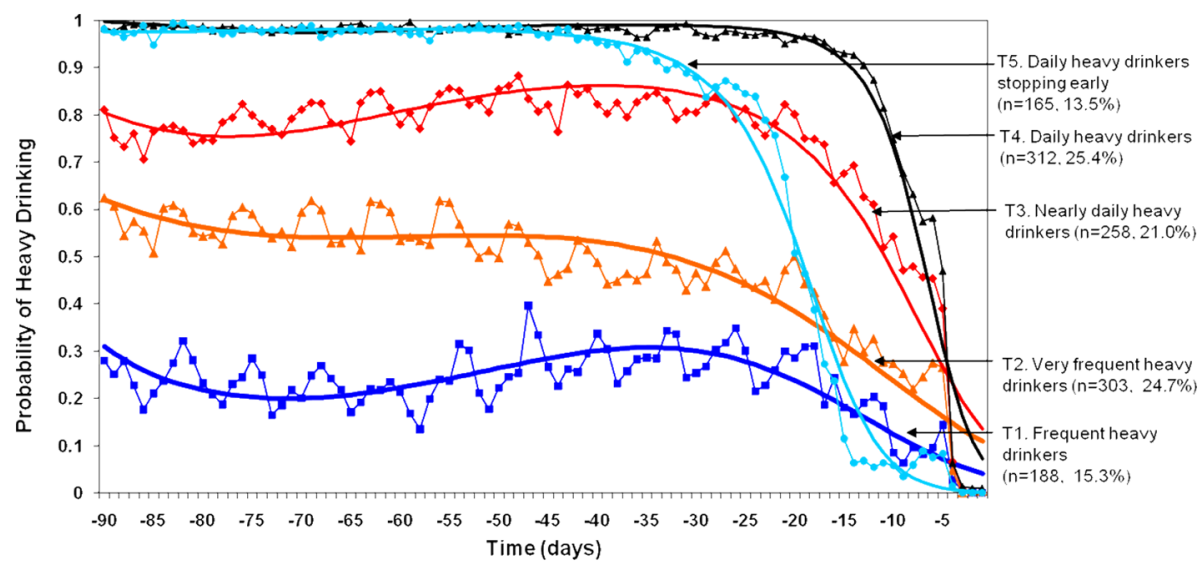

Figure 1.

Five baseline trajectories of heavy drinking. ${ }^{\text {a }}$

a Solid lines with symbols represent sample-based probabilities of drinking based on all subjects weighted by the posterior probability of trajectory membership. Solid lines without symbols represent model-based probabilities of drinking over time for each trajectory group. 
T5. Daily drinkers stopping early $(n=198,16.2 \%)$

T4. Consistent daily drinkers ( $n=354.28 .9 \%$ )

政

T3. Nearly daily drinkers ( $n=219.17 .9 \%$ )

莺

खे

2. Very frequent drinkers $(n=259.21 .1 \%)$

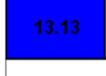

\begin{tabular}{l|l|l}
8.59 & 7.07 & 9.6
\end{tabular} \begin{tabular}{|l|l|}
\hline 4.8 & 9.6
\end{tabular}

7.12

T1. Frequent drinkers ( $n=196.16 .0 \%)$

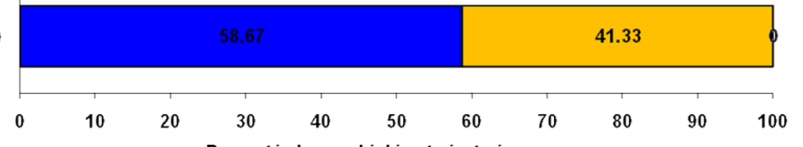

Percent in heavy drinking trajectories

Figure 2.

Cross-classification in baseline any drinking and baseline heavy drinking trajectories. 


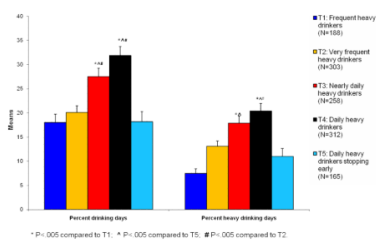

Figure 3.

Continuous outcomes by heavy drinking trajectories. 


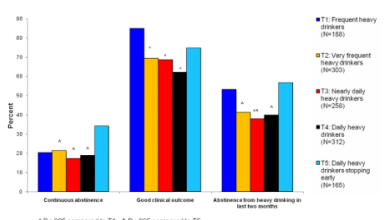

Figure 4.

Categorical outcomes by heavy drinking trajectories. 


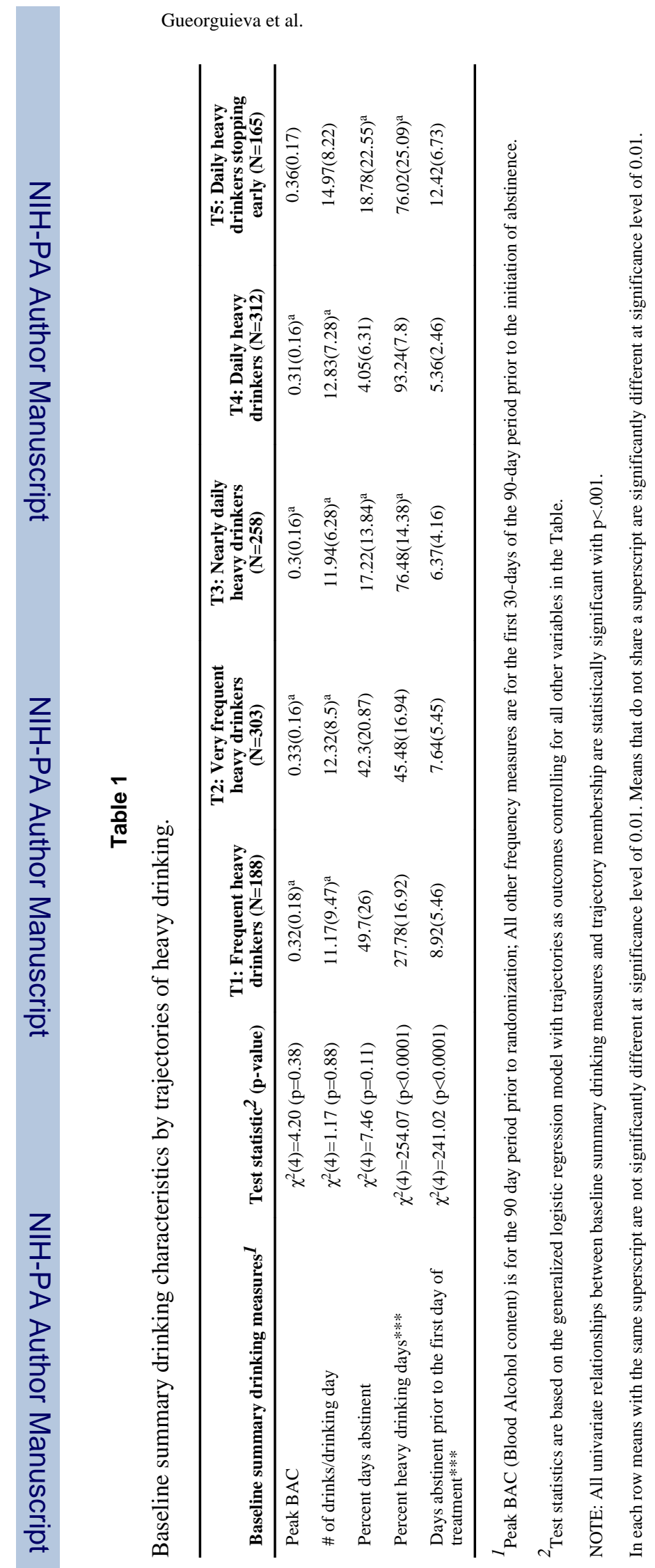

Alcohol. Author manuscript; available in PMC 2013 March 1. 


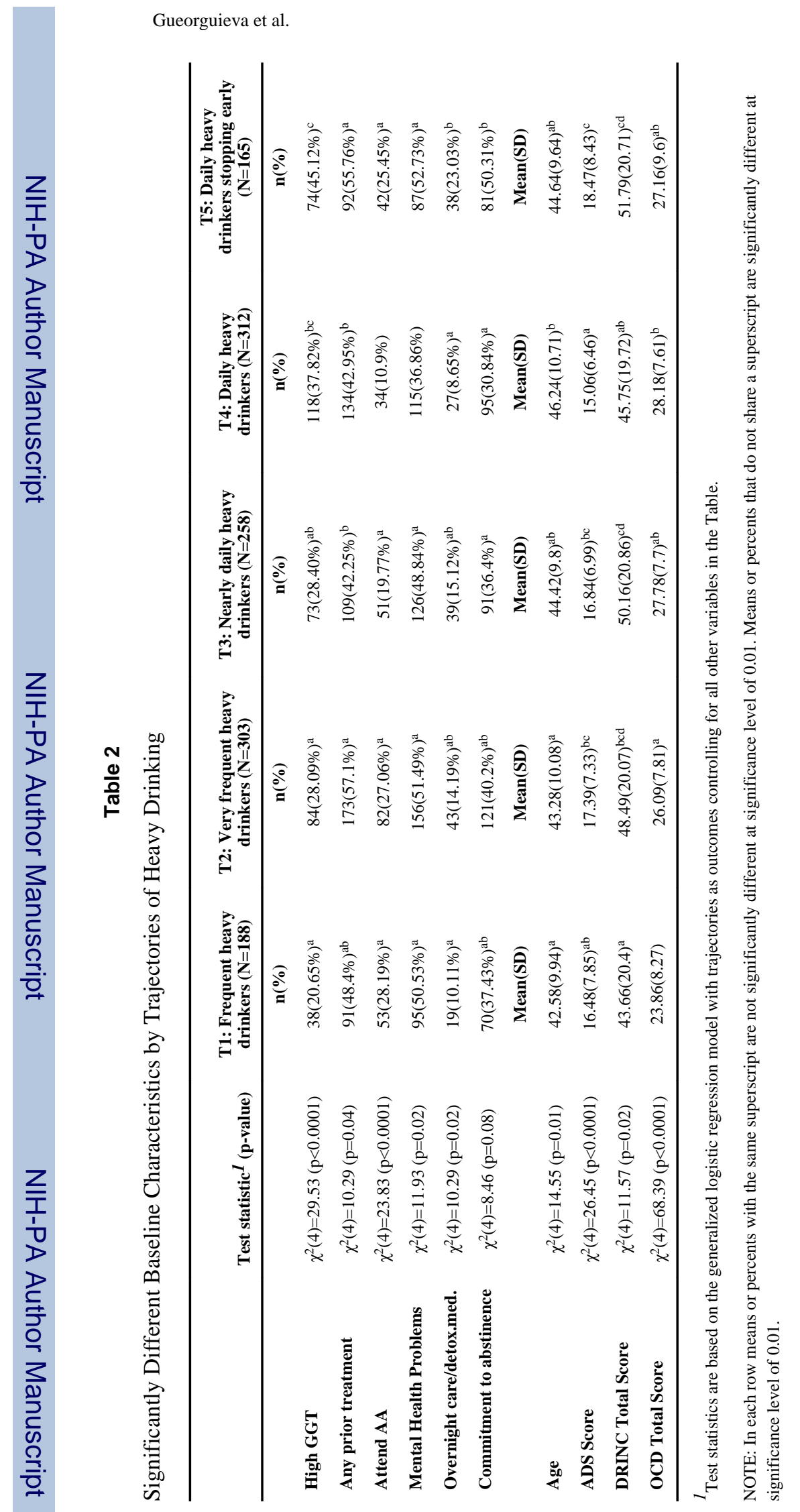

Alcohol. Author manuscript; available in PMC 2013 March 1. 